Copyright ( 2013 IEEE. Personal use of this material is permitted. Permission from IEEE must be obtained for all other uses, in any current or future media, including reprinting/republishing this material for advertising or promotional purposes, creating new collective works, for resale or redistribution to servers or lists, or reuse of any copyrighted component of this work in other works. 


\title{
Modelling the PDF Evolution in a CMOS Inverter during Switching
}

\author{
R. M. Howard \\ School of Electrical Engineering and Computing \\ Curtin University \\ GPO Box U1987, Perth, Western Australia, 6845, Australia.
}

\begin{abstract}
A model which facilitates the simulation of switching in a CMOS inverter, and a model for the associated PDF evolution, are proposed. The latter model is based on a Gaussian basis set. Simulation results, for the additive Gaussian white noise case, show, first, that the Gaussian assumption for the probability density function of the output jitter is valid at high input noise levels but with deviations from the Gaussian form for the propagation delay. Second, the output noise variance at a set time varies and is a maximum close to the midpoint between the high and low states. Third, the rms jitter of the output signal increases with the input rise time and the output load capacitance but decreases with increasing noise bandwidth. Fourth, the high correlation between the input and output signals leads to lower propagation delay jitter than expected.
\end{abstract}

Keywords--PDF evolution, switching, jitter, CMOS inverter.

\section{INTRODUCTION}

The random phenomena inherent in the physical world typically limits the performance of engineered entities and, accordingly, significant research interest has been undertaken to understand and characterize their origins and effects. The operation of many systems depends on switching is some form and such an operation is subject to jitter. The paper by Stanley [1] provides good motivation for the study of the nature of switching. In many engineering papers, e.g. [2], [3], jitter is assumed to have a Gaussian probability density function (PDF). This assumption is consistent, for example, with Gaussian noise input to a memoryless system and where the rms noise level is consistent with a linear range of operation and the probability of multiple level crossings is low. In general, however, due to the non-linear nature of switching, jitter values are not expected to be consistent with a Gaussian PDF, e.g. [4]. Accordingly, it is of interest to establish the noise level at which the Gaussian assumption breaks down as the transition to a non-Gaussian PDF is likely to lead to higher output rms noise and jitter levels than expected.

The first contribution of this paper is to investigate the Gaussian assumption of jitter arising in the prototypical case of switching in a CMOS inverter. This is facilitated by establishing a suitable differentiable approximation to the piecewise defined current-voltage characteristics of the NMOS and PMOS devices and an overall differentiable input-output relationship. The input noise is modelled consistent with finite bandwidth white noise and, overall, the CMOS inverter model is that of a Hammerstein system with feedback which is subject to finite bandwidth white noise. The second contribution of this paper is to use a Gaussian based orthonormal basis set of functions, along with an interpolation function, to model the probability density function evolution associated with switching in a CMOS inverter. This approach is straight- forward in comparison with alternative approaches, e.g. [5], which require, for example, the solution of a non-linear partial differential equation followed by high order integration. Finally, simulation results show, first, that the Gaussian assumption for jitter is valid up until high noise levels. Second, the effect of input rise time, noise bandwidth and load capacitance on the output noise and jitter. Third, the existence of high correlation between the input and output signals which leads to lower than expected propagation delay jitter.

\section{BACKGROUND}

Consider an orthonormal basis set $\left\{b_{1}, \ldots, b_{i}, \ldots\right\}$ defined on the interval where the probability density function of interest is non-zero. With such a basis set, the probability density function of a random variable $X$ can be written as [6]

$$
f_{X}(x)=c_{1} b_{1}(x)+c_{2} b_{2}(x)+\ldots
$$

where the ith coefficient is

$$
c_{i}=\boldsymbol{E}\left[b_{i}(X)\right]=\int_{-\infty}^{\infty} b_{i}(x) f_{X}(x) d x
$$

Here $\boldsymbol{E}$ is the expectation operator. The coefficient $c_{i}$ can be estimated from $N$ independent samples, $x_{1}, \ldots, x_{N}$, according to

$$
\hat{c}_{i}=\frac{1}{N} \sum_{i=1}^{N} b_{i}\left(x_{i}\right)
$$

and, consequently, an estimator for $f_{X}(x)$ is

$$
\hat{f}_{X}(x)=\hat{c}_{1} b_{1}(x)+\hat{c}_{2} b_{2}(x)+\ldots
$$

It is the case, [6], that the estimate of the ith coefficient, as given by (3), is unbiased and has a variance which decreases according to $1 / N$.

\section{A. Gaussian Based Basis Set}

For $i \in\{0,1, \ldots\}$ it is the case that

$$
\int_{-\infty}^{\infty} \frac{e^{-x^{2} / \sigma^{2}}}{2 \pi \sigma^{2}} B_{i}(x) B_{j}(x) d x=\left\{\begin{array}{lr}
0 & i \neq j \\
1 & i=j
\end{array}\right.
$$


where

\section{MODELLING CMOS INVERTER}

$$
B_{i}(x)=\frac{\pi^{1 / 4} \sqrt{\sigma} H_{i}(x / \sigma)}{2^{(i-1) / 2} \sqrt{i !}}
$$

Here $H_{i}$ is the $i t h$ Hermite polynomial defined by

$$
H_{i}(x)=2 x H_{i-1}(x)-2(i-1) H_{i-2}(x)
$$

where $H_{0}(x)=1$ and $H_{1}(x)=2 x$. It then follows that

$$
\left\{\begin{array}{l}
b_{0}(x)=\sqrt{2} \pi^{1 / 4} \sqrt{\sigma} H_{0}\left[\frac{x-\mu}{\sigma}\right] \cdot \frac{e^{-(x-\mu)^{2} / 2 \sigma^{2}}}{\sqrt{2 \pi} \sigma}, \ldots, \\
b_{i}(x)=\frac{\pi^{1 / 4} \sqrt{\sigma} H_{i}[(x-\mu) / \sigma]}{2^{(i-1) / 2} \sqrt{i !}} \cdot \frac{e^{-(x-\mu)^{2} / 2 \sigma^{2}}}{\sqrt{2 \pi} \sigma}, \ldots
\end{array}\right\}
$$

is an orthogonal basis set for $(-\infty, \infty)$ based on Gaussian functions with a mean $\mu$ and a variance $\sigma^{2}$.

\section{B. PDF Evolution}

The PDF at a specific time $t$ can be modelled, consistent with (1), using the mean $\mu(t)$, the variance $\sigma^{2}(t)$ and the Gaussian basis set defined in (8), according to

$$
f_{X(t)}(x)=c_{0}(t) b_{0}(x, t)+\ldots+c_{i}(t) b_{i}(x, t)+\ldots
$$

where $c_{i}(t)=\boldsymbol{E}\left[b_{i}(X(t), t)\right]$.

From samples of the random process taken at the times $\Delta t, 2 \Delta t, \ldots$, the mean, variance and basis set coefficients can be estimated. An interpolating function $r$, with $r(0)=1$ and $r(k)=0, k \in Z, k \neq 0$, can then be used to establish approximate values for all other times:

$$
\begin{gathered}
\mu(t) \approx \sum_{k=1}^{\infty} \mu(k \Delta t) r\left(\frac{t-k \Delta t}{\Delta t}\right) \\
\sigma^{2}(t) \approx \sum_{k=1}^{\infty} \sigma^{2}(k \Delta t) r\left(\frac{t-k \Delta t}{\Delta t}\right) \\
c_{i}(t) \approx \sum_{k=1}^{\infty} c_{i}(k \Delta t) r\left(\frac{t-k \Delta t}{\Delta t}\right)
\end{gathered}
$$

In a similar manner the PDF evolution with time can be approximated according to

$$
f_{X(t)}(x) \approx \sum_{k=1}^{\infty} r\left(\frac{t-k \Delta t}{\Delta t}\right) \sum_{i=0}^{\infty} c_{i}(k \Delta t) b_{i}(x, k \Delta t)
$$

An estimator for this PDF can be established from sample values and estimates of $\mu(k \Delta t), \sigma^{2}(k \Delta t)$ and $c_{i}(k \Delta t)$.
The structure of a CMOS inverter, and its associated circuit model, are detailed in Fig. 1. The model assumes, as is typically the case, that the internal MOSFET capacitance are small relative to the load capacitance and can be included in this capacitance. The current sources in the model have, as a first order approximation, the following definitions:

$$
i_{N}=0, v_{G S} \leq V_{T} \quad i_{P}=0, v_{S G} \leq V_{T}
$$

$$
\begin{aligned}
& i_{N}=\left\{\begin{array}{l}
k_{n}\left[\left(v_{G S}-V_{T}\right) v_{D S}-\frac{v_{D S}^{2}}{2}\right] \\
\frac{k_{n}}{2}\left(v_{G S}-V_{T}\right)^{2}
\end{array}\right. \\
& \text { triode region } \\
& v_{D S} \leq v_{G S}-V_{T} \\
& \text { saturation region } \\
& v_{D S}>v_{G S}-V_{T} \\
& i_{P}=\left\{\begin{array}{l}
k_{p}\left[\left(v_{S G}-V_{T}\right) v_{S D}-\frac{v_{S D}^{2}}{2}\right] \\
\frac{k_{p}}{2}\left(v_{S G}-V_{T}\right)^{2}
\end{array}\right.
\end{aligned}
$$

where $V_{T}$ is the threshold voltage, $k_{n}=\mu_{n} C_{o x} W / L$, $k_{p}=\mu_{p} C_{o x} W / L$, and $C_{o x}=\varepsilon_{r} \varepsilon_{o} / t_{o x}$. Here $\mu_{n}$ and $\mu_{p}$ are, respectively, the surface mobility of electrons and holes, $\varepsilon_{r}$ is the relative permittivity of the oxide material, $\varepsilon_{o}$ is the permittivity of free space and $t_{o x}$ is the oxide thickness. The output voltage of the CMOS inverter is the solution of the first order non-linear differential equation:

$$
C_{L} \frac{\mathrm{d}}{\mathrm{d} t} v_{o}(t)=i_{P}\left(v_{i}, v_{o}, t\right)-i_{N}\left(v_{i}, v_{o}, t\right)
$$

This equation is consistent with a Hammerstein system with feedback.

\section{A. Modelling to Facilitate Simulation}

To facilitate numerical solution of (17), the currents, first, can be modelled according to:
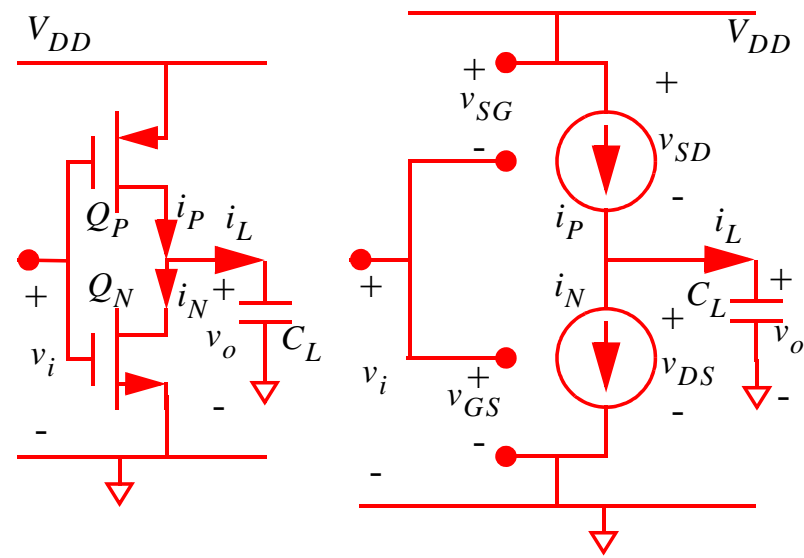

Fig. 1. Left: Structure of a CMOS inverter. Right: Model for CMOS inverter. 


$$
\begin{gathered}
i_{N}\left(v_{G S}, v_{D S}\right)=k_{n}\left[\left(v_{G S}-V_{T}\right) v_{D S}-\frac{v_{D S}^{2}}{2}\right] \cdot u\left[v_{G S}-V_{T}\right] \cdot \\
u\left[\left(v_{G S}-V_{T}\right)-v_{D S}\right]+ \\
\frac{k_{n}}{2}\left(V_{G S}-V_{T}\right)^{2} \cdot u\left[v_{G S}-V_{T}\right] \cdot u\left[v_{D S}-\left(v_{G S}-V_{T}\right)\right] \\
i_{P}\left(v_{S G}, v_{S D}\right)=k_{p}\left[\left(v_{S G}-V_{T}\right) v_{S D}-\frac{v_{S D}^{2}}{2}\right] \cdot u\left[v_{S G}-V_{T}\right] \cdot \\
u\left[\left(v_{S G}-V_{T}\right)-v_{S D}\right]+ \\
\frac{k_{p}}{2}\left(V_{S G}-V_{T}\right)^{2} \cdot u\left[v_{S G}-V_{T}\right] \cdot u\left[v_{S D}-\left(v_{S G}-V_{T}\right)\right]
\end{gathered}
$$

where $u$ is the unit step function. Second, to avoid the nondifferentiability of these equations at the transition points between the cutoff, triode and saturation regions, a useful approach is to approximate the unit step function by the hyperbolic tangent function according to

$$
u(x) \approx 0.5[1+\tanh (x / \Delta)]
$$

for a suitably small value of $\Delta$. The resulting differentiable functions approximate the original piecewise defined functions arbitrarily closely and, hence, facilitate the solution of (17).

\section{B. Modelling Input Signal}

The input signal, for the case of switching from high to low with a fall time of $t_{R}$ seconds, is modelled according to

$$
v_{i}(t)=\frac{V_{D D}}{2}\left[1-\tanh \left(\left(t-t_{R}\right) / \tau\right)\right]+n(t)
$$

where $n$ is the additive noise and the constant $\tau$ is defined according to $\tau=t_{R} / 2 \operatorname{atanh}(0.8)$.

\section{Modelling Noise}

The input noise signal $n$ is one signal from a random process defining signals with a Gaussian PDF, a flat spectrum on the interval $\left[0, f_{\max }\right]$, a rms amplitude of $A_{o}$ and defined according to

$$
n(t)=\sum_{k=1}^{m} \frac{\sqrt{2} A_{o}}{\sqrt{m}} \sin \left(2 \pi f_{k} t+\phi_{k}\right)
$$

where $\phi_{1}, \ldots, \phi_{m}$ and $f_{1}, \ldots, f_{m}$ are outcomes of independent random variables that define, respectively, the phase and frequency of the $m$ sinusoids comprising the signal. The random variables defining the phase are assumed to have a uniform distribution on $[-\pi, \pi]$ and $f_{1}, \ldots, f_{m}$ are outcomes of a Poisson point process with a rate $\lambda=m_{o} / f_{\text {max }}$ yielding, on average, $m_{o}$ frequencies randomly selected from the interval $\left[0, f_{\max }\right]$. A specific outcome of this point process yields $m$ frequencies in the interval $\left[0, f_{\max }\right]$.

\section{RESULTS}

The following parameter values are assumed: $V_{D D}=5 \mathrm{~V}, k_{n}=k_{p}=0.01, V_{T}=1 \mathrm{~V}, C_{L}=10 \mathrm{pF}$, $f_{\max }=10^{9} \mathrm{~Hz}, \quad \Delta=0.1, \quad \Delta t=0.1 \mathrm{~ns}, \quad t_{R}=1 \mathrm{~ns}$, $m_{o}=1000, A_{o}=0.1$. Results are obtained using 10,000 independent signals. The rms output noise levels detailed below are at the level of $V_{D D} / 2$ and all rms jitter levels assume the first passage time across this same level.

One input signal, and its associated output signal, are shown in Fig. 2. The PDFs of the level crossing time to $V_{D D} / 2$, for the input and output signals, and the PDF of the propagation delay, are shown in Fig. 3. The PDF evolution is shown in Fig. 4 using the model specified by (10) to (13).

\section{A. Gaussianity and Correlatedness}

As is evident in Fig. 3, the PDF of the level crossing times, and the propagation delay, are approximately Gaussian but with some deviation from the Gaussian form at low levels for the propagation delay. When the rms input noise level is at a high level of $A_{o}=0.25$, the propagation delay shows significant deviation from the Gaussian form. For set times the PDF of the output signal is close to Gaussian but with a divergence from the Gaussian form as the mean level diverges from the mid-level of $V_{D D} / 2$.

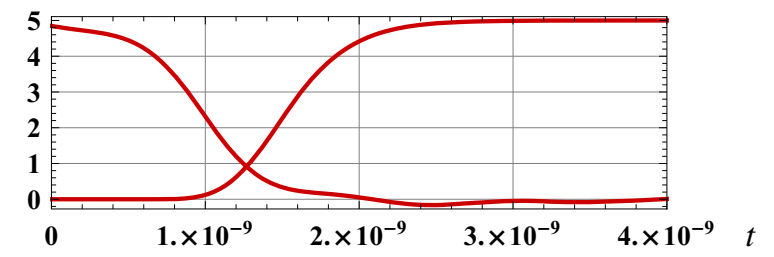

Fig. 2. Example of an input and output signal.

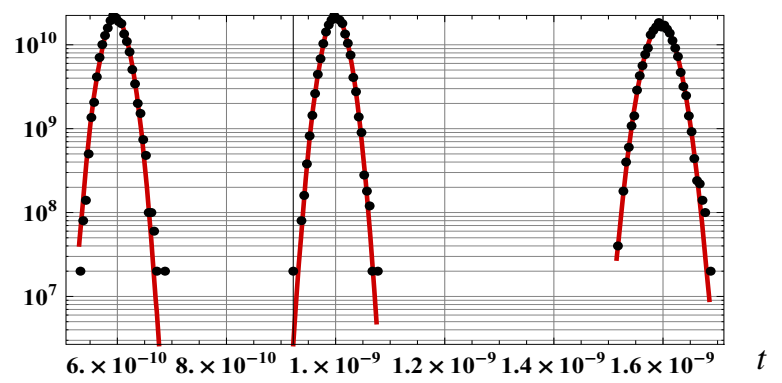

Fig. 3. PDF of level crossing times to the level $V_{D D} / 2$ for input signal (middle), output signal (right) and PDF for propagation delay (left). The smooth curve is a Gaussian function. The dots are simulation values. 


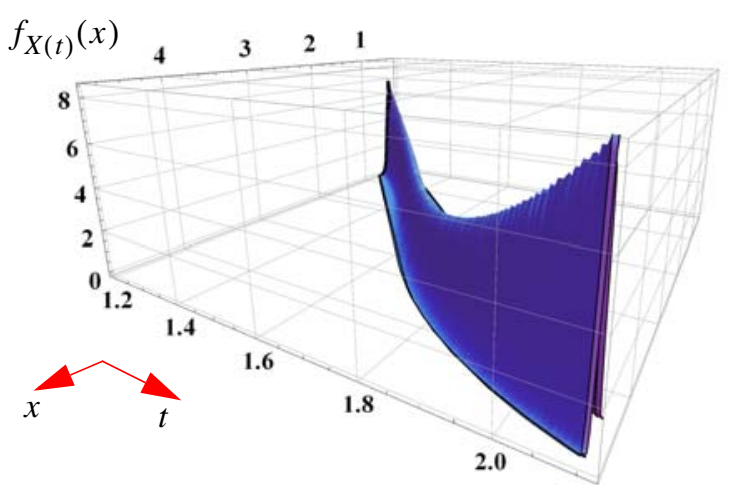

Fig. 4. PDF evolution of output signal. Time is in ns. 20 coefficients in the basis function expansion have been used along with a triangular interpolation function: $r(t)=[1-|t|][u(t+1)-u(t-1)]$.

The rms jitter levels of the input, output and propagation delay, respectively, are $18.1 p s, 21.2 p s$ and $18.8 p s$. The fact that the propagation delay jitter is smaller than the output jitter is evidence of the input and output noise being correlated at the time when the output is $V_{D D} / 2$. The correlation coefficient is -0.58 and is relatively insensitive to the input rms noise level.

\section{B. Effect of Input Rise Time}

The output rise time, output rms noise level, output jitter, and the propagation delay jitter, are specified in Table 1 for the above defined parameters but with a varying input rise time. A model for the output rms jitter (jitter in $p s$, rise time in $n s$ ) is

$$
\text { output rms jitter }=13.4+9.1 t_{R}-0.085 t_{R}^{2}
$$

The results detailed in Table show the importance of a fast rise time, and circuitry to facilitate this, in minimizing the output rms noise and the output rms jitter.

\section{Effect of Threshold on Jitter \& RMS Noise Level}

Consistent with the probability density function evolution shown in Fig. 4, the output rms noise level is a maximum close to $V_{D D} / 2$. The signal to noise ratio improves with increasing level and is around $60 \%$ higher at a level of $3 \mathrm{~V}$

Table 1. Output rise time, rms noise, rms jitter and propagation delay jitter.

\begin{tabular}{lllll}
\hline $\begin{array}{l}\text { Input rise } \\
\text { time }\end{array}$ & $\begin{array}{l}\text { Output } \\
\text { rise time }\end{array}$ & $\begin{array}{l}\text { Output } \\
\text { rms noise }\end{array}$ & $\begin{array}{l}\text { Output } \\
\text { rms jitter }\end{array}$ & $\begin{array}{l}\text { Propagation } \\
\text { delay jitter }\end{array}$ \\
\hline $0.25 \mathrm{~ns}$ & $0.75 \mathrm{~ns}$ & $0.106 \mathrm{~V}$ & $15.5 \mathrm{ps}$ & $12.4 \mathrm{ps}$ \\
$0.5 \mathrm{~ns}$ & $0.79 \mathrm{~ns}$ & $0.118 \mathrm{~V}$ & $17.7 \mathrm{ps}$ & $13.0 \mathrm{ps}$ \\
$1 \mathrm{~ns}$ & $0.89 \mathrm{~ns}$ & $0.134 \mathrm{~V}$ & $22.4 \mathrm{ps}$ & $18.8 \mathrm{ps}$ \\
$2 \mathrm{~ns}$ & $1.12 \mathrm{~ns}$ & $0.157 \mathrm{~V}$ & $31.8 \mathrm{ps}$ & $36.4 \mathrm{ps}$ \\
$5 \mathrm{~ns}$ & $1.65 \mathrm{~ns}$ & $0.196 \mathrm{~V}$ & $56.9 \mathrm{ps}$ & $90.7 \mathrm{ps}$ \\
$10 \mathrm{~ns}$ & $2.4 \mathrm{~ns}$ & $0.237 \mathrm{~V}$ & $96 \mathrm{ps}$ & $173 \mathrm{ps}$ \\
$20 \mathrm{~ns}$ & $3.6 \mathrm{~ns}$ & $0.280 \mathrm{~V}$ & $162 \mathrm{ps}$ & $318 \mathrm{ps}$ \\
\hline
\end{tabular}

than at $2.5 \mathrm{~V}$. The rms jitter, however, increases from $22.4 p s$ to $23.3 p s$.

\section{Effect of Noise Bandwidth}

For the same rms input noise level, the effective filtering of high frequency noise leads to a lower output rms noise level $\left(134 \mathrm{mV}\right.$ for $f_{\max }=1 \mathrm{GHz}, 99 \mathrm{mV}$ for $\left.f_{\text {max }}=2 \mathrm{GHz}\right)$, and lower output jitter (22.4ps for $f_{\max }=1 \mathrm{GHz}, 16.4 \mathrm{ps}$ for $f_{\max }=2 \mathrm{GHz}$ ). The lower the bandwidth of the noise, the greater the level of input-output noise correlation $\left(-0.58\right.$ for $f_{\max }=1 \mathrm{GHz},-0.34$ for $f_{\max }=2 \mathrm{GHz}$ ) due to the decrease in the level of noise filtering.

\section{E. Effect of CMOS Load Capacitance}

The output rms jitter, and the propagation delay jitter, increase as the load capacitance increases. The output rms jitter changes from $20.1 p s$ when $C_{L}=5 p F$ to $27.3 p s$ when $C_{L}=20 p F$. Whilst increasing load capacitance provides greater noise filtering it results in a slower rise time of the output signal which is the cause of the increased jitter. The rms noise level decreases with increasing capacitance consistent with the increased noise filtering.

\section{CONCLUSION}

Using a model which facilitates simulation of switching, and a Gaussian basis set which underpins a model for PDF evolution, simulation results have shown, first, that the Gaussian assumption for the probability density function of the output jitter is valid at high input noise levels but this is not the case for the propagation delay. Second, the output noise variance at a set time varies and is a maximum close to $V_{D D} / 2$. Third, the rms jitter of the output signal increases with the input rise time and the output load capacitance but decreases with increasing noise bandwidth. Fourth, the high correlation between the input and output signals leads to lower propagation delay jitter than expected.

\section{REFERENCES}

[1] H. E. Stanley et. al., 'Correlated randomness and switching phenomena’, Physica A, vol. 389, 2010, pp. 2880-2893.

[2] M. Lohning \& G. Fettweis, 'The effects of aperture jitter and clock jitter in wideband ADCs', Computer Standards and Interfaces, vol. 29, 2007, pp 11-18.

[3] J. Fatome, et.al., 'All-optical measurement of background, amplitude and timing jitters for high speed pulse trains or prbs sequences using autocorrelation function', Optical Fibre Technology, vol. 14, 2008, pp. 84-91.

[4] Y. Madec \& C. Japhet, 'First passage time problem for a drifted Ornstein-Uhlenbeck process', Mathematical Biosciences, vol. 189, 2004, pp. 131-140.

[5] J. Li et. al., 'Advances of the probability density evolution method for nonlinear stochastic systems', Probabilistic Engineering Mechanics, vol. 28, 2012, pp. 132-142.

[6] S. C. Schwartz, 'Estimation of probability density by an orthogonal series', The Annals of Mathematical Statistics, vol. 38, 1967, pp. 1261-1265. 